\title{
Long non-coding RNA MEG3 suppresses the growth of glioma cells by regulating the miR-96-5p/MTSS1 signaling pathway
}

\author{
SHOUDAN ZHANG and WENSHI GUO \\ Department of Neurosurgery, The First Affiliated Hospital of Jinzhou Medical University, \\ Jinzhou, Liaoning 121001, P.R. China
}

Received April 29, 2019; Accepted August 12, 2019

DOI: $10.3892 / \mathrm{mmr} .2019 .10659$

\begin{abstract}
Glioma is one of the most common types of tumor of the central nervous system with high mobility and mortality. The prognosis of patients with high-grade glioma is poor. Therefore, it is urgent to develop the therapeutic strategies for the treatment of glioma. Long non-coding RNAs (lncRNAs) have been reported as potential inducers or suppressors of numerous types of tumors including glioma. Previous studies have revealed that IncRNA maternally expressed gene 3 (MEG3) is involved in the initiation and progression of cancer; however, the underlying mechanisms remain unclear. In the present study, MEG3 was downregulated in glioma tissue. In addition, downregulation of MEG3 was observed in human glioma cell lines compared with normal astrocyte cells. Furthermore, overexpressed MEG3 inhibited the proliferation, migration and invasion of glioma cells. Additionally, microRNA-96-5p (miR-96-5p) was a promising target of MEG3, and the promoting effects of miR-96-5p on cell growth and metastasis could be reversed by upregulated MEG3. Metastasis suppressor 1 (MTSS1) was predicted as the putative target of miR-96-5p, and its expression was restored by MEG3. In summary, the present data provided novel insight into the roles of MEG3 in glioma, and MEG3/miR-96-5p/MTSS1 signaling could be a promising therapeutic target for the treatment of patients with glioma.
\end{abstract}

\section{Introduction}

Glioma is one of the most prevalent and aggressive malignancies of the brain, characterized with unfavorable prognosis. It accounts for $\sim 30-40 \%$ of primary tumors and $\sim 80 \%$ of malignant neoplasia of the central nervous system (1). Although the conventional treatments have been substantially improved by

Correspondence to: Dr Wenshi Guo, Department of Neurosurgery, The First Affiliated Hospital of Jinzhou Medical University, 2 Renmin Road, Guta, Jinzhou, Liaoning 121001, P.R. China

E-mail: wenshi_guo@163.com

Key words: glioma, long non-coding RNA MEG3, proliferation, migration, invasion adjuvant radiotherapy, targeted biological therapy and chemotherapy, the overall survival rate of patients with glioma is still poor $(2,3)$, thus the development of promising therapeutic strategies against glioma is urgent.

Long non-coding RNAs (lncRNAs) are a new group of non-protein-coding RNAs with $>200$ nucleotides (4-6). Emerging evidence has indicated that lncRNAs are involved in the initiation and progression of tumors including glioma, and they function as promoters or suppressors in tumorigenesis by regulating cancer cell proliferation, differentiation, migration and invasion (7-15). Furthermore, aberrant levels of lncRNAs have been reported in tumor cells, indicating the potential roles of lncRNAs in cancer (14-18).

LncRNA maternally expressed gene 3 (MEG3) is a novel tumor suppressor located on chromosome $14 \mathrm{q} 32$, and previous studies have revealed the impaired expression of MEG3 in a variety of cancers $(10,15,17)$. MEG3 was revealed to inhibit the initiation and development of tumors including glioma through numerous signaling pathways (19-23). In addition, previous results have indicated that MEG3 is a promising biomarker and prognostic indicator of glioma (17); however, the underlying mechanism and potential molecular targets of MEG3 in glioma have not been completely elucidated.

MicroRNAs (miRNAs) are a class of non-coding RNAs and are $\sim 22 \mathrm{nt}$ in length, which are putative downstream targets of IncRNAs $(24,25)$. It is well established that miRNAs exert their functions by binding to the 3 ' untranslated region of corresponding mRNAs (26-29). The levels of miRNAs are disrupted in cancer, consequently resulting in tumor development $(30,31)$. For example, upregulation of $\mathrm{H} 19$ was detected in colorectal cancer, which could promote cell proliferation via miR-675 (9). In addition, miR-93 was able to induce the proliferation of glioma cells by targeting the phosphatidylinositol 3 kinase/protein kinase B (PI3K/AKT) pathway (26). Furthermore, miR-140 and miR-152 interacted with lncRNAs in glioma $(32,33)$; however, the effects of miRNAs in glioma remain elusive.

In the present study, the effects of MEG3-regulated signaling on the growth and metastasis of glioma cells were investigated, and MEG3 was downregulated in glioma tissues and cells. Overexpressed MEG3 inhibited cell proliferation, migration and invasion in vitro, and miR-96-5p was a promising target of MEG3. Overexpression of MEG3 abolished the effects of miR-93/metastasis suppressor 1 (MTSS1) signaling 
on facilitating the growth and metastasis of glioma cells. Collectively, these findings elucidated the roles of MEG3 in the progression of glioma, providing new insight for the treatment of this disease.

\section{Materials and methods}

Clinical samples. A total of 30 paired human glioma and para-carcinoma tissues were obtained from patients (14 males and 16 females; aged 35-62 years old) that underwent surgical resection at The First Affiliated Hospital of Jinzhou Medical University (Jinzhou, China) between August 2016 and May 2018. None of the patients had received other treatments prior to surgery. The study was conducted in accordance with the Helsinki declaration and was approved by the Institutional Review Board of The First Affiliated Hospital of Jinzhou Medical University. Written informed consent was signed by each patient. Metastasis was detected in 11 cases, and 18 patients were diagnosed with grade I or II glioma. All specimens were immediately snap-frozen using liquid nitrogen and stored at $-80^{\circ} \mathrm{C}$ until RNA extraction.

Cell culture. Three human glioma cell lines (GSC11, M059J and D54) and normal human astrocyte cell line (A735) were purchased from the American Type Culture Collection (Manassas, VA, USA). Cells were cultured in Dulbecco's modified Eagle's medium (DMEM) supplemented with 10\% fetal bovine serum (FBS), $100 \mathrm{U} / \mathrm{ml}$ penicillin and $100 \mu \mathrm{g} / \mathrm{ml}$ streptomycin (HyClone; GE Healthcare Life Sciences), and maintained at $37^{\circ} \mathrm{C}$ in a humidified $5 \% \mathrm{CO}_{2}$ atmosphere.

Cell transfection. To generate the MEG3-knockdown model, short hairpin RNA (shRNA) sequences targeting MEG3 (sh-MEG3) and negative control (sh-NC) were purchased from Shanghai GenePharma Co., Ltd. After annealing, shRNA was integrated into lentiviral pU6-Luc-Puro vector (Shanghai GenePharma Co., Ltd.). To establish the MEG3 overexpression model, wild-type (o/e-MEG3) or mutant (o/e-NC) MEG3 fragment was amplified by PCR and subcloned into pcDNA3.1 vector (Invitrogen; Thermo Fisher Scientific, Inc.). Glioma cells were transfected with recombinant lentiviral vectors or the controls. Up- or downregulation of MEG3 was confirmed using RT-qPCR. The mimic or inhibitor of miR-96-5p and the corresponding negative control (NC) were purchased Shanghai GenePharma Co., Ltd., and were transfected into glioma cells using Lipofectamine ${ }^{\circledR} 2000$ (Invitrogen; Thermo Fisher Scientific, Inc.). At 8-h post-transfection, the culture medium was replenished with fresh DMEM containing 10\% FBS.

Reverse transcription-quantitative polymerase chain reaction $(R T-q P C R)$. RT-qPCR was used to evaluate the expression levels of MEG3, miR-96-5p and MTSS1. miRNA was extracted using miRNeasy Mini Kit (Qiagen, Inc.). TaqMan MicroRNA Assay kit (Applied Biosystems; Thermo Fisher Scientific, Inc.) was used to evaluate the expression of miR-96-5p, and qPCR was carried out using Applied Biosystem 7500 Real-Time PCR system. Total RNA from clinical samples or cell lines was extracted using TRIzol ${ }^{\circledR}$ reagent (Invitrogen; Thermo Fisher Scientific, Inc.) according to the manufacturer's protocols. The concentration of eluted RNA was determined by NanoDrop 1000 spectrophotometer (Thermo Fisher Scientific, Inc.). cDNA was synthesized using a PrimeScript ${ }^{\mathrm{TM}}$ RT kit (Takara Biotechnology Co., Ltd.), and qPCR was performed using SYBR Green PCR Master Mix (Takara Biotechnology Co., Ltd.) according to the manufacturer's protocols. Endogenous GAPDH and U6 small nuclear RNA were used as internal controls for mRNA and miRNA, respectively. The sequences of forward and reverse primers were as follows, respectively: MEG3, 5'-GCCCTAGGGGAGTGACTACA-3' and 5'-ACT CGGGACATACCTGCTCT-3'; miR-96-5p, 5'-UUUGGCACU AGCACAUUUUUGC-3' and 5'-UUCGGCACUAGCACA UUUUUGC-3'; MTSS1, 5'-TCAAGA ACAGATGGAAGA ATGG-3' and 5'-TGCGGTAGCGGTAATGTG-3'; GAPDH, 5'-GCAAGAGCACAAGAGGAAGA-3' and 5'-ACTGTG AGGAGGGGAGATTC-3'; and U6, 5'-CTCGCTTCGGCA GCACATA-3' and 5'AACGATTCACGAATTTGCGT-3'. The PCR program used for the thermocycler was $95^{\circ} \mathrm{C}$ for $5 \mathrm{~min}$, followed by 45 cycles at $95^{\circ} \mathrm{C}$ for $15 \mathrm{sec}, 60^{\circ} \mathrm{C}$ for $20 \mathrm{sec}$ and $72^{\circ} \mathrm{C}$ for $10 \mathrm{sec}$. Relative expression levels were calculated using $2^{-\Delta \Delta C q}$ method (34).

Western blotting. Total protein was extracted from tissues or cells using radioimmunoprecipitation assay buffer (Beyotime Institute of Biotechnology). Protein concentration was evaluated by bicinchoninic acid assay (Beyotime Institute of Biotechnology). Equal amounts $(30 \mu \mathrm{g})$ of protein samples were separated using 10\% SDS-PAGE gel and then transferred onto PVDF membranes (EMD Millipore). The membranes were blocked in tris-buffered saline (TBS) with 5\% skimmed milk for $2 \mathrm{~h}$ at room temperature, and then were incubated with primary antibodies: MTSS1 (1:500; cat. no. sc-101204) or GAPDH (1:1,000; cat. no. sc-47724; both from Santa Cruz Biotechnology Inc.) at $4^{\circ} \mathrm{C}$ overnight. The membranes were then incubated with corresponding horseradish peroxidase-conjugated secondary antibody $(1: 5,000$; cat. no. sc-2371; Santa Cruz Biotechnology Inc.) for $1 \mathrm{~h}$ at $37^{\circ} \mathrm{C}$, followed by visualization using an enhanced chemiluminescence protein detection kit (Pierce Biotechnology; Thermo Fisher Scientific, Inc). Protein bands were quantified by densitometric analysis using ImageJ software (NIH).

Cell proliferation assay. Cells were harvested $24 \mathrm{~h}$ post-transfection, and $1 \times 10^{4}$ cells were seeded in 96-well plates. Cell proliferation was evaluated using Cell Counting Kit-8 (CCK-8; Dojindo Molecular Technologies, Inc.) at days 1, 2, 3 and 4 post-inoculation. Briefly, $10 \mu \mathrm{l}$ of CCK-8 solution was added into each well at each time-point. After incubation at $37^{\circ} \mathrm{C}$ for an another $2 \mathrm{~h}$, the absorbance at $450 \mathrm{~nm}$ was determined by a microplate reader (Bio-Rad Laboratories, Inc.).

Transwell assay. Cell migration and invasion were determined by a Transwell assay. For the migration assay, a total of $1 \times 10^{5}$ cells were diluted in FBS-free medium and seeded into the upper chamber (BD Biosciences). For the invasion assay, cells were added onto a Matrigel ${ }^{\circledR}$-pre-coated (Sigma-Aldrich; Merck KGaA) upper chamber. Subsequently, $500 \mu 1$ of culture medium containing $10 \%$ FBS was inoculated into the lower chamber. After overnight incubation, non-migratory/-invasive cells were detached using a cotton swab, while the 
A
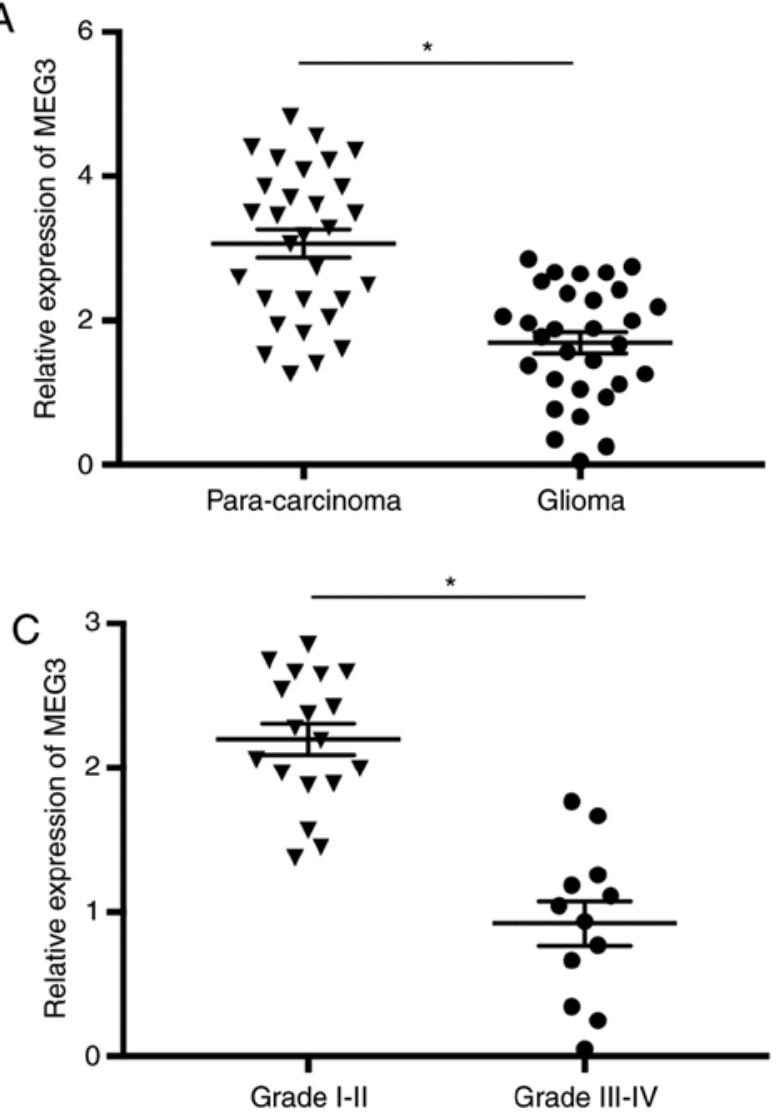
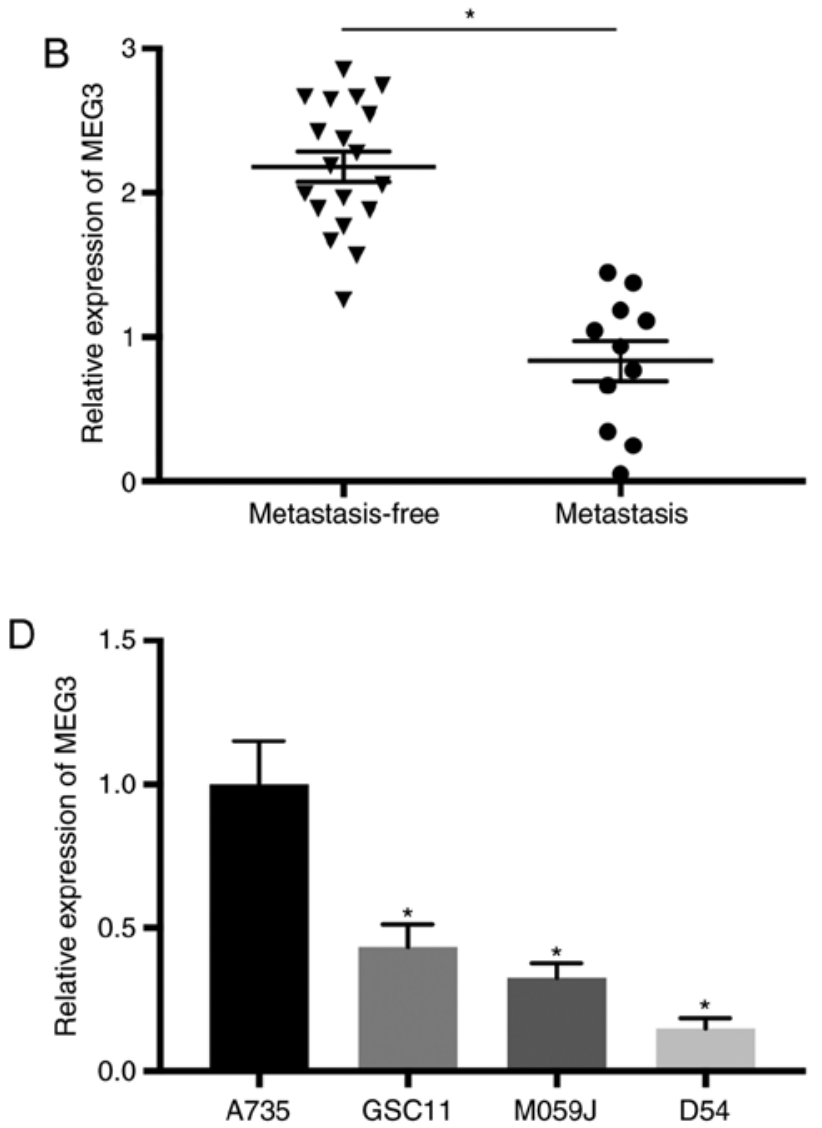

Figure 1. MEG3 is downregulated in glioma tissues and cells. (A) The expression level of MEG3 was examined in 30 glioma tissues and matched para-carcinoma controls by RT-qPCR. (B) MEG3 expression was evaluated in glioma patients with metastasis compared with the controls. (C) The level of MEG3 was determined in glioma tissues with various stages. (D) The expression level of MEG3 was assessed in normal human astrocyte cell line (A735) and glioma cells (GSC11, M059J and D54). "P<0.05. MEG3, maternally expressed 3; RT-qPCR, reverse transcription-quantitative polymerase chain reaction.

migrated/invaded cells in the lower chamber were fixed in $4 \%$ paraformaldehyde and stained using $0.5 \%$ crystal violet. The number of migratory/invasive cells were counted in five randomly selected fields using an inverted light microscope (magnification, x200; Olympus Corporation).

Northern blot analysis. Total RNA was extracted using TRIzol ${ }^{\circledR}$ reagent (Invitrogen; Thermo Fisher Scientific, Inc.) according to the manufacturer's protocols. Equal amounts of RNA $(20 \mu \mathrm{g})$ were loaded onto $15 \%$ TBE-urea gels and separated using a $15 \%$ urea-PAGE gel, then transferred onto positively charged nylon membranes (GE Healthcare Life Sciences) and cross-linked by UV irradiation. The blots were hybridized using DIG-labelled probe for miR-96-5p (Exiqon A/S) overnight at $42^{\circ} \mathrm{C}$. Subsequently, the membranes were rinsed using low-stringency buffer (2X SSC containing $0.1 \%$ SDS). The levels of miRNAs were determined by a DIG Luminescent Detection Kit (Roche Diagnostics). U6 RNA was used as a loading control.

Bioinformatic prediction and luciferase activity assay. Targetscan (www.targetscan.org/) and miRanda (www. microrna.org/microrna/) were used to predict the potential targets of MEG3 or miR-96-5p. Wild-type (WT) fragments of the 3'UTR of MEG3/MTSS1 with putative binding sites of miR-96-5p were purchased from Shanghai GenePharma Co.,
Ltd., and cloned into pmirGLO Dual-Luciferase miRNA Target Expression Vector (Promega Corporation) according to the manufacturer's protocols. MEG3/MSS1-3'UTR-MUT reporter containing mutant miR-96-5p binding sites was used and generated using QuikChange Multi Site-Directed Mutagenesis Kit (Stratagene, La Jolla, CA, USA). Subsequently, the recombinant vectors were co-transfected with miR-NC or miR-96-5p mimics into DH5 $\alpha$ competent cells. Luciferase activity was evaluated $48 \mathrm{~h}$ post-transfection by Dual Luciferase Reporter Assay System (Promega Corporation) according to the manufacturer's protocols, and firefly luciferase activity was normalized to that of Renilla luciferase.

Statistical analysis. Data are presented as the means \pm standard deviation and analyzed using SPSS 17.0 (SPSS, Inc.). The significance of differences in groups was analyzed using Student's t-test or one-way analysis of variance (ANOVA). A student-Newman-Keuls test was performed following ANOVA. The association between RNA levels was evaluated using Spearman's correlation analysis. $\mathrm{P}<0.05$ was considered to indicate a statistically significant difference.

\section{Results}

MEG3 is downregulated in glioma tissues/cells and associated with poor prognosis. The levels of MEG3 were evaluated in 
A

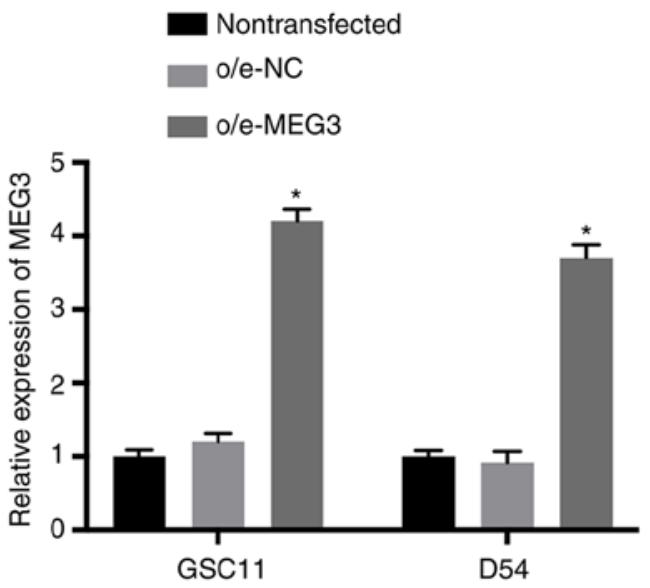

B

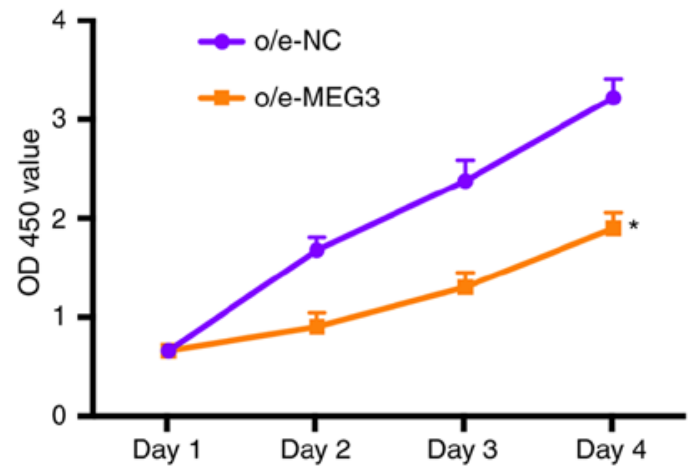

D

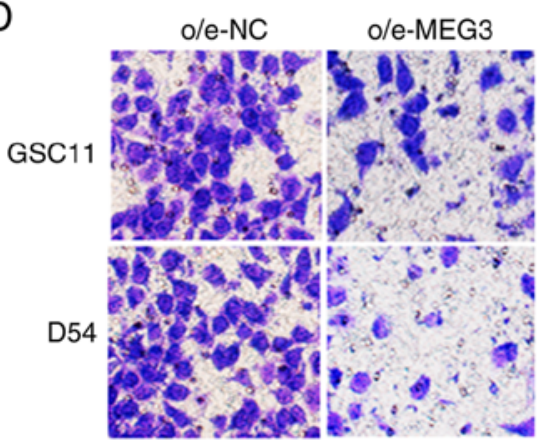

$\mathrm{F}$

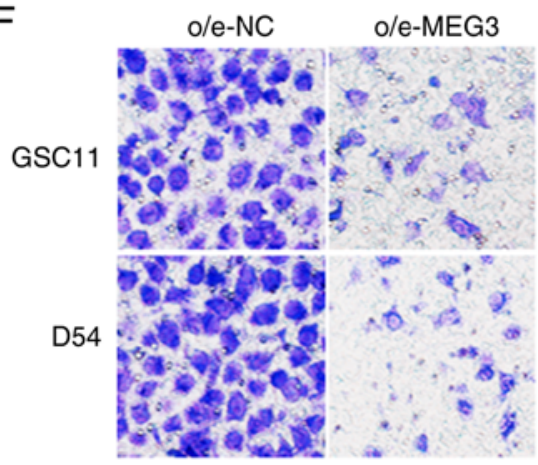

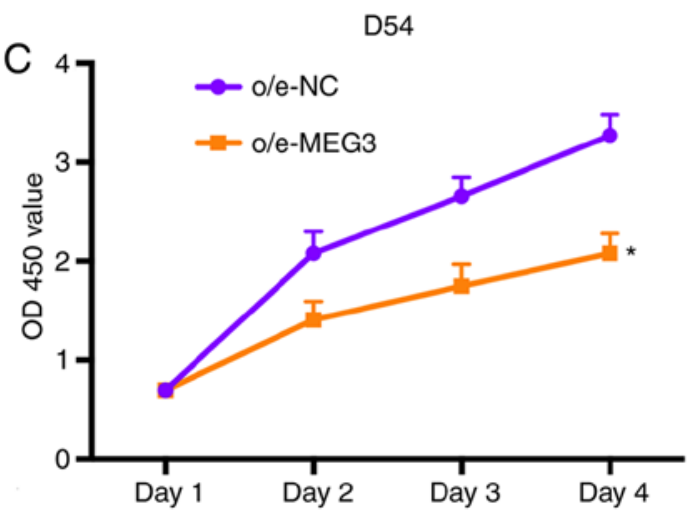

$E$

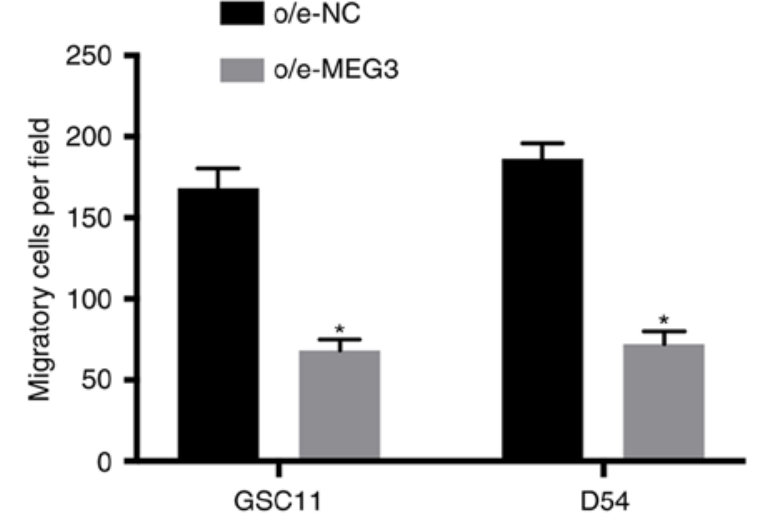

G

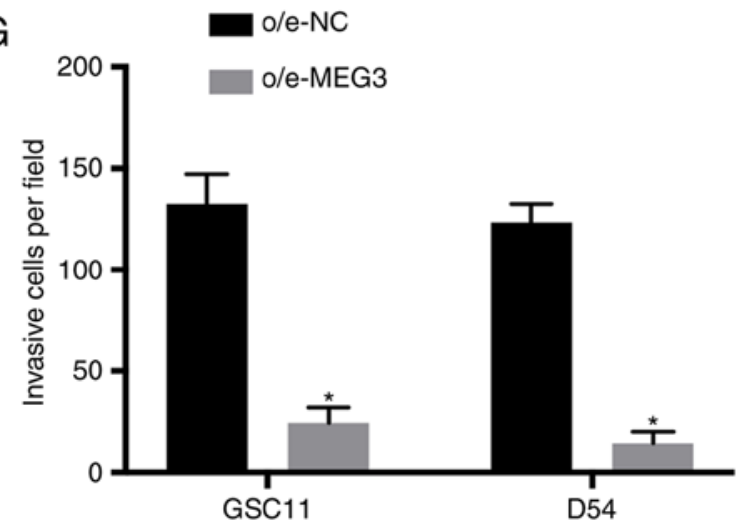

Figure 2. Upregulated MEG3 suppresses the proliferation, migration and invasion of glioma cells. (A) Transfection efficiency of o/e-MEG3 was determined by RT-qPCR. (B and C) The proliferative activities of GSC11 and D54 cells transfected with o/e- MEG3 or o/e-NC were evaluated using CCK-8 assay. (D and E) The migration of transfected GSC11 and D54 cells were evaluated using a Transwell assay (magnification, $x 200$ ). (F and G) The invasion of GSC11 and D54 cells transfected with o/e-MEG3 or o/e-NC were determined (magnification, x200). "P<0.05. MEG3, maternally expressed 3; RT-qPCR, reverse transcription-quantitative polymerase chain reaction; CCK-8, Cell Counting Kit-8; NC, negative control. 
A WT-MEG3 5'-GAAUGCCAAAGA-3' miR-96-5p 3'-AUCACGGUUUAG-5'

MUT-MEG3 5'-GAACAUACGCGA-3'

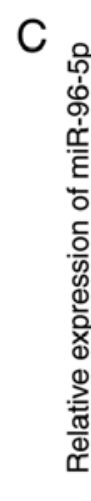
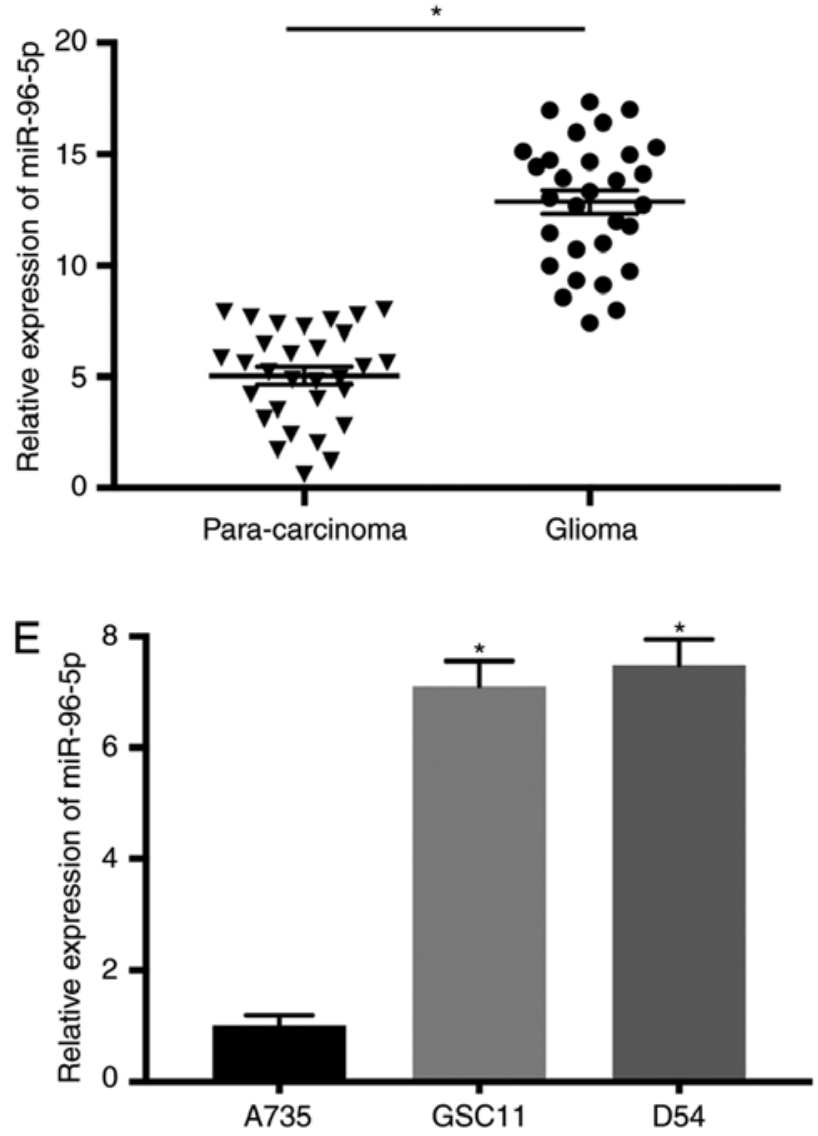

B

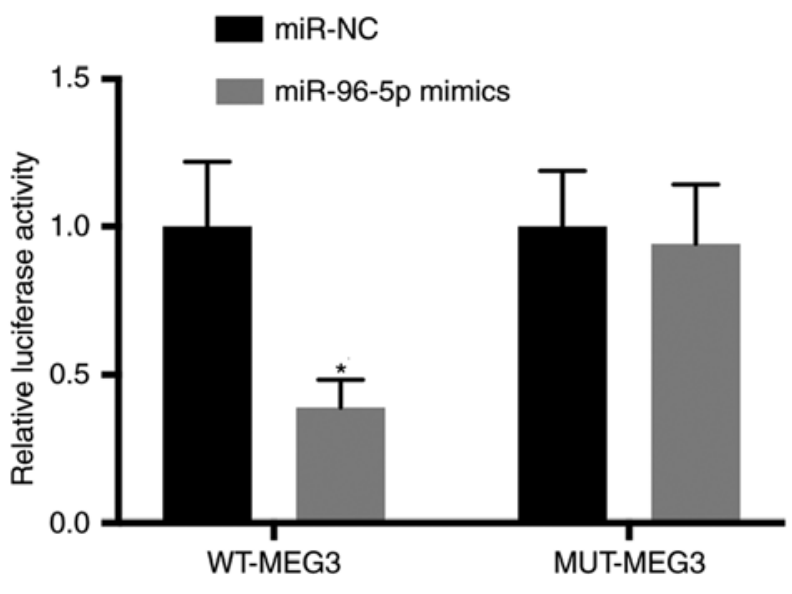

$\mathrm{D}$

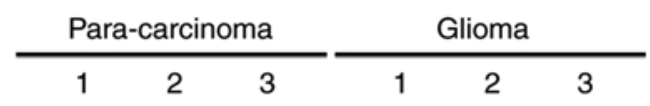

miR-96-5p

U6

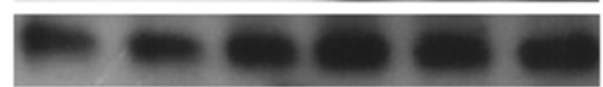

$\mathrm{F}$ miR-96-5p

U6

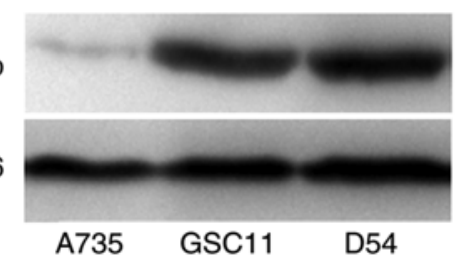

Figure 3. miR-96-5p is the potential target of MEG3 in glioma. (A) The putative binding sites of miR-96-5p on MEG3 transcript. (B) Overexpression of miR-96-5p resulted in significantly reduced luciferase activity of MEG3-WT, while no change was observed in MEG3-MUT. (C and D) The expression level of miR-96-5p was upregulated in glioma tissues. (E and F) The level of miR-96-5p in GSC11 and D54 cells was increased. * $<<0.05$. miR, microRNA; MEG3, maternally expressed 3; WT, wild-type; MUT, mutant.

30 glioma and matched para-carcinoma samples by RT-qPCR The results indicated that MEG3 was significantly downregulated in glioma tissues compared with the non-tumor controls (Fig. 1A). In addition, the association between MEG3 expression and the progression of glioma was investigated. The results revealed that MEG3 was significantly decreased in glioma patients with metastasis compared with the controls (Fig. 1B). Furthermore, the expression level of MEG3 was significantly reduced in aggressive glioma (Fig. 1C), suggesting that downregulation of MEG3 is associated with the development of glioma. Additionally, significant decrease of MEG3 was revealed in glioma cells compared with normal human astrocytes ( $\mathrm{P}<0.05$ vs. A735; Fig. 1D). Collectively, the expression of MEG3 was downregulated in glioma, which could contribute to the metastasis and tumor progression in patients.

Overexpression of MEG3 inhibits the proliferation, migration and invasion of glioma cells. To explore the effects of MEG3 on the growth and metastasis of glioma cells, MEG3 was overexpressed in GSC11 and D54 cells. The transfection efficiency was determined using RT-qPCR ( $\mathrm{P}<0.05$ vs. nontransfected; Fig. 2A). Furthermore, the results of CCK-8 assay indicated that the proliferation of GSC11 and D54 cells transfected with o/e-MEG3 was inhibited compared with the control 

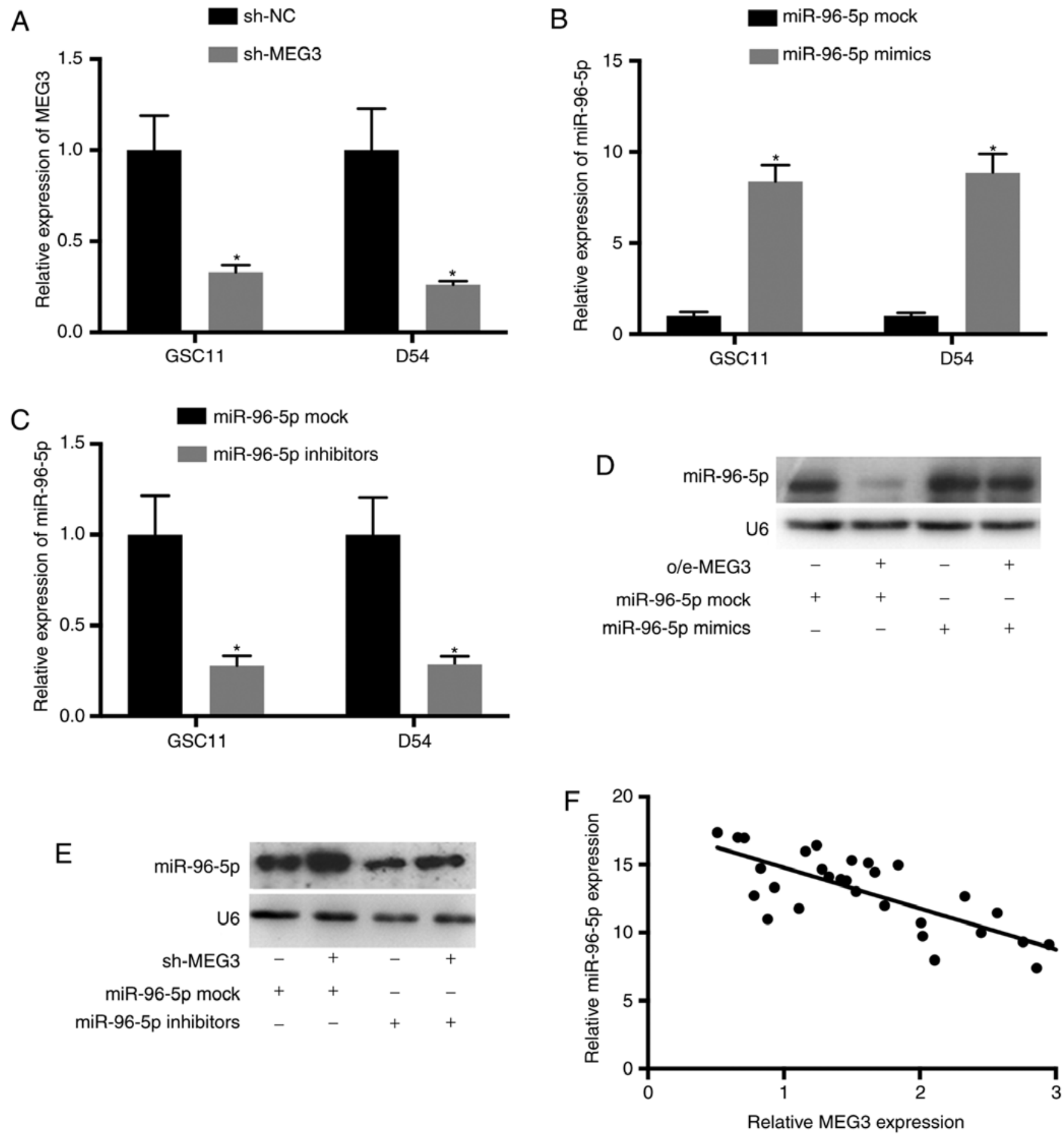

Figure 4. The expression level of miR-96-5p is downregulated by MEG3. (A-C) GSC11 and D54 cells were transfected with sh-MEG3, miR-96-5p mimics or inhibitors. (D) The upregulation of miR-96-5p in GSC11 cells transfected with miR-96-5p mimics was abolished by o/e-MEG3. (E) The downregulation of miR-96-5p in cells transfected with miR-96-5p inhibitors was reversed by sh-MEG3. (F) Spearman's correlation analysis revealed the negative correlation between miR-96-5p and MEG3 in glioma tissues ( $\mathrm{r}=-0.2281 ; \mathrm{P}=0.00712) .{ }^{*} \mathrm{P}<0.05$. miR, microRNA; MEG3, maternally expressed 3.

(Fig. 2B and C). In addition, Transwell assay revealed that the migratory and invasive abilities of o/e-MEG3-transfected glioma cells were significantly suppressed (Fig. 2D-G). These findings indicated that the growth and metastasis of glioma could be inhibited by overexpressed MEG3.

miR-96-5p is the potential target of MEG3 in glioma. To investigate whether MEG3 is a putative tumor suppressor in glioma and functions by targeting its downstream miRNAs, the complementary binding sites between miR-96-5p and MEG3 were predicted through bioinformatics analysis using LncBase Predicted v.2 (Fig. 3A). The relationship of MEG3 and miR-96-5p was further confirmed by luciferase assay. Luciferase reporters carrying wild-type (MEG3-WT) and mutant (MEG3-MUT) sequence of predicted miR-96-5p binding sites were constructed. The results indicated that overexpression of miR-96-5p significantly reduced the activity of luciferase plasmid containing MEG3-WT compared with the 
A
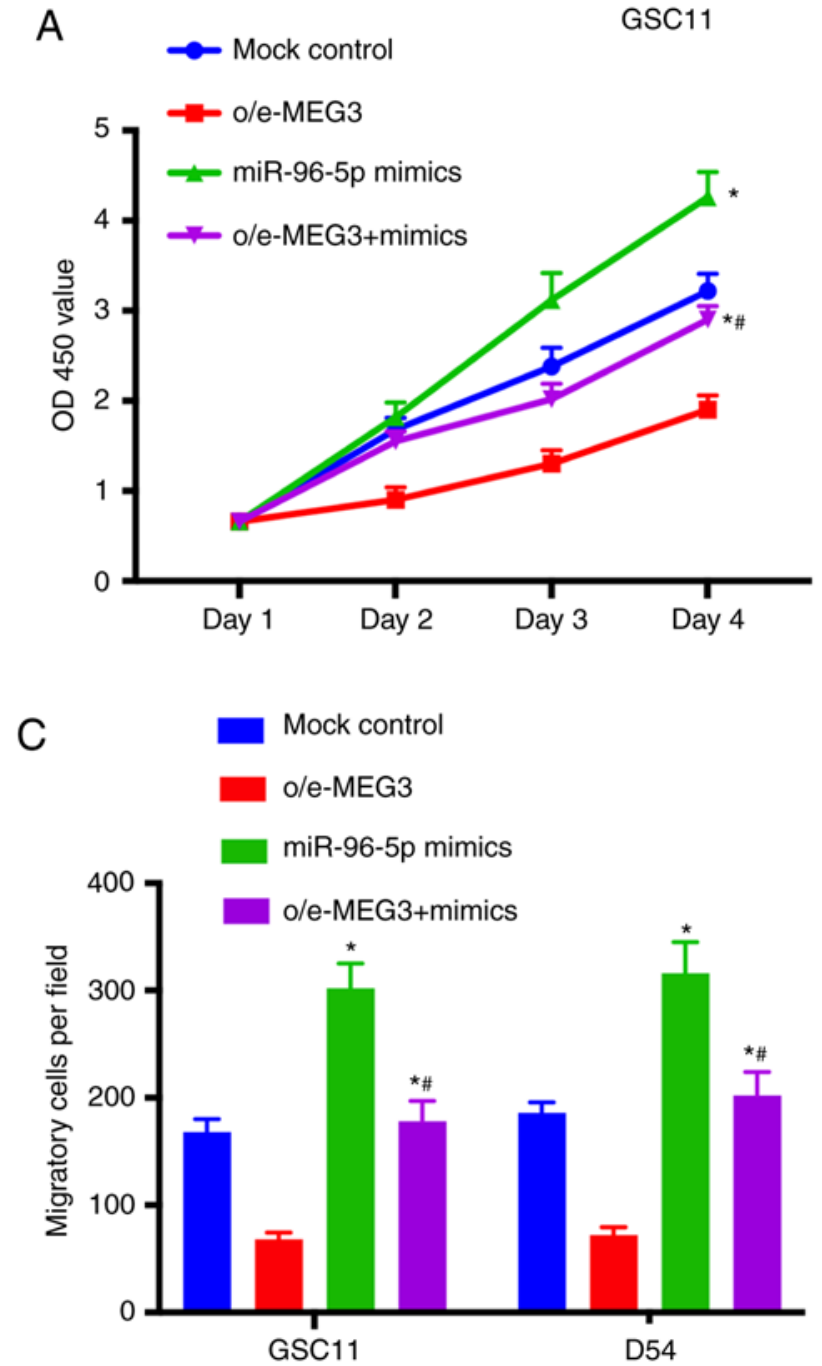

B
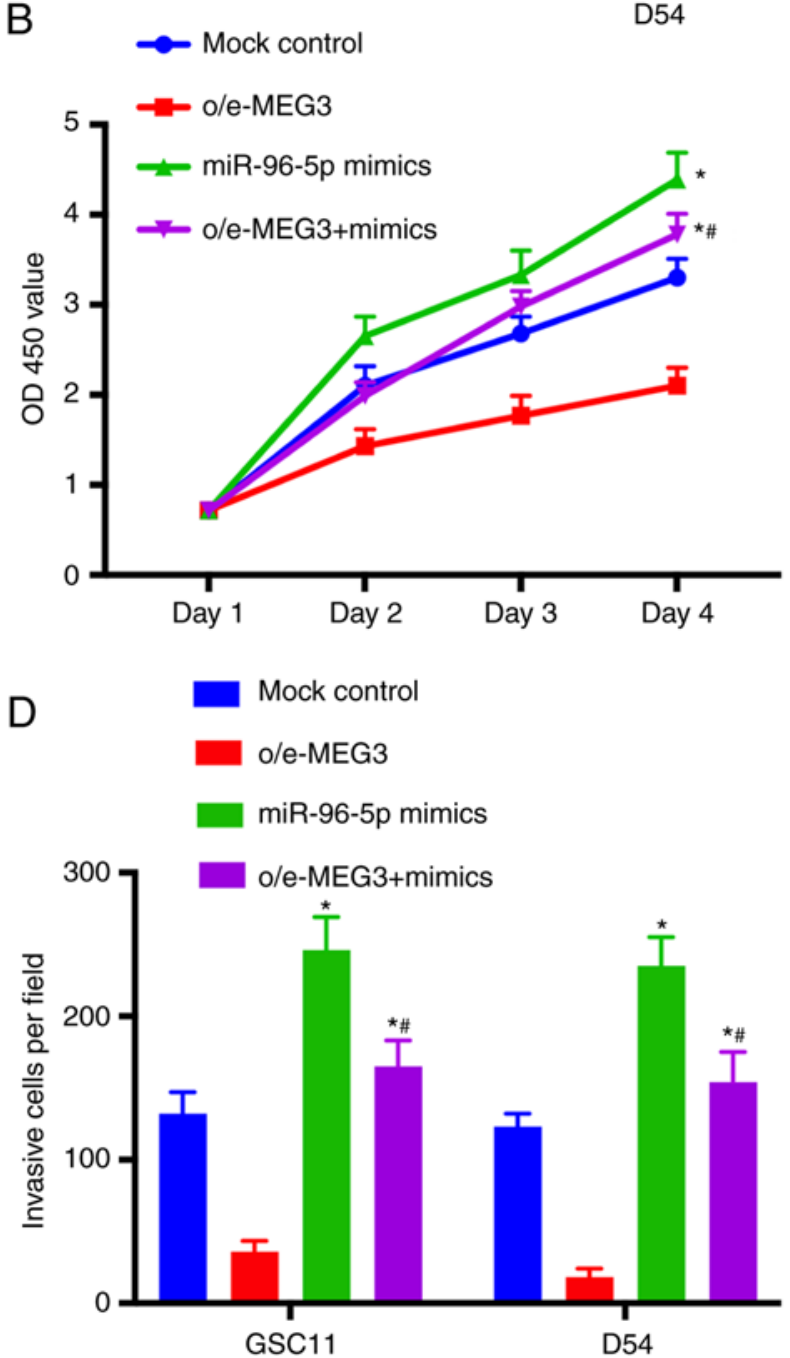

Figure 5. miR-96-5p promotes the proliferation, migration and invasion of glioma cells. (A and B) The proliferation of GSC11 and D54 cells transfected with mock control, o/e-MEG3, miR-96-5p mimics or co-transfected with o/e-MEG3 and miR-96-5p mimics was determined using CCK-8. (C) The migration of transfected glioma cells was determined using a Transwell assay. (D) The invasive activities of GSC11 and D54 cells was increased after the treatment with miR-96-5p mimics. * $\mathrm{P}<0.05$ vs. the mock control. ${ }^{\#} \mathrm{P}<0.05$ vs. o/e-MEG3. miR, microRNA; MEG3, maternally expressed 3; CCK-8, Cell Counting Kit-8.

control (Fig. 3B). Furthermore, the results of RT-qPCR and northern blotting indicated that miR-96-5p was upregulated in glioma tissues (Fig. 3C and D). Additionally, upregulation of miR-96-5p was revealed in GSC11 and D54 cells as revealed by RT-qPCR and northern blotting $(\mathrm{P}<0.05$ vs. A735; Fig. 3E and F).

In order to further investigate the effects of MEG3 on miR-96-5p expression, glioma cells were co-transfected with o/e-MEG3 and miR-96-5p mimics, or with sh-MEG3 and miR-96-5p inhibitors respectively. The transfection efficiencies were evaluated using RT-qPCR $(\mathrm{P}<0.05$ vs. nontransfected; Fig. 4A-C). Northern blotting indicated that the upregulation of miR-96-5p in GSC11 cells transfected with miR-96-5p mimics was reversed by adding o/e-MEG3 (Fig. 4D). Conversely, the downregulation of miR-96-5p in GSC11 cells transfected with miR-96-5p inhibitors was abolished after co-transfection with sh-MEG3 (Fig. 4E). Additionally, the association between MEG3 and miR-96-5p was determined using Spearman's correlation analysis. The results indicated that the expression levels of miR-96-5p and MEG3 in glioma tissues were inversely correlated (Fig. 4F).
miR-96-5p is involved in the growth of glioma cells. The effects of miR-96-5p on the growth and metastasis of glioma were further evaluated. The results of CCK- 8 assay indicated that cell proliferation was significantly promoted in the miR-96-5p mimics group (Fig. 5A and B). In addition, the migratory abilities of GSC11 and D54 cells were increased after the treatment with miR-96-5p mimics (Fig. 5C). Furthermore, the invasion of glioma cells was promoted by miR-96-5p mimics (Fig. 5D). In summary, these findings indicated that miR-96-5p was able to promote the growth and metastasis of glioma.

MTSS1 is the putative target of miR-96-5p. To identify novel downstream molecules, the complementary sequence of miR-96-5p in MTSS1 transcripts was predicted (Fig. 6A). The interaction between MEG3 and miR-96-5p was further confirmed by luciferase assay. Luciferase reporter vectors containing the wild-type (MTSS1-WT) and mutant (MTSS1-MUT) sequence of predicted miR-96-5p binding sites were constructed. The results revealed that overexpressed miR-96-5p significantly decreased the activity of luciferase plasmids carrying the wild-type MEG3 sequence 
A WT-MTSS15-UUGAUUUUUCUGAAGGUGCCAAA-3

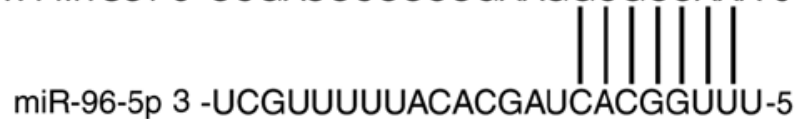

MUT-MTSS1 5 -UUGAUUUUUCUGAAGCACGGUUU-3
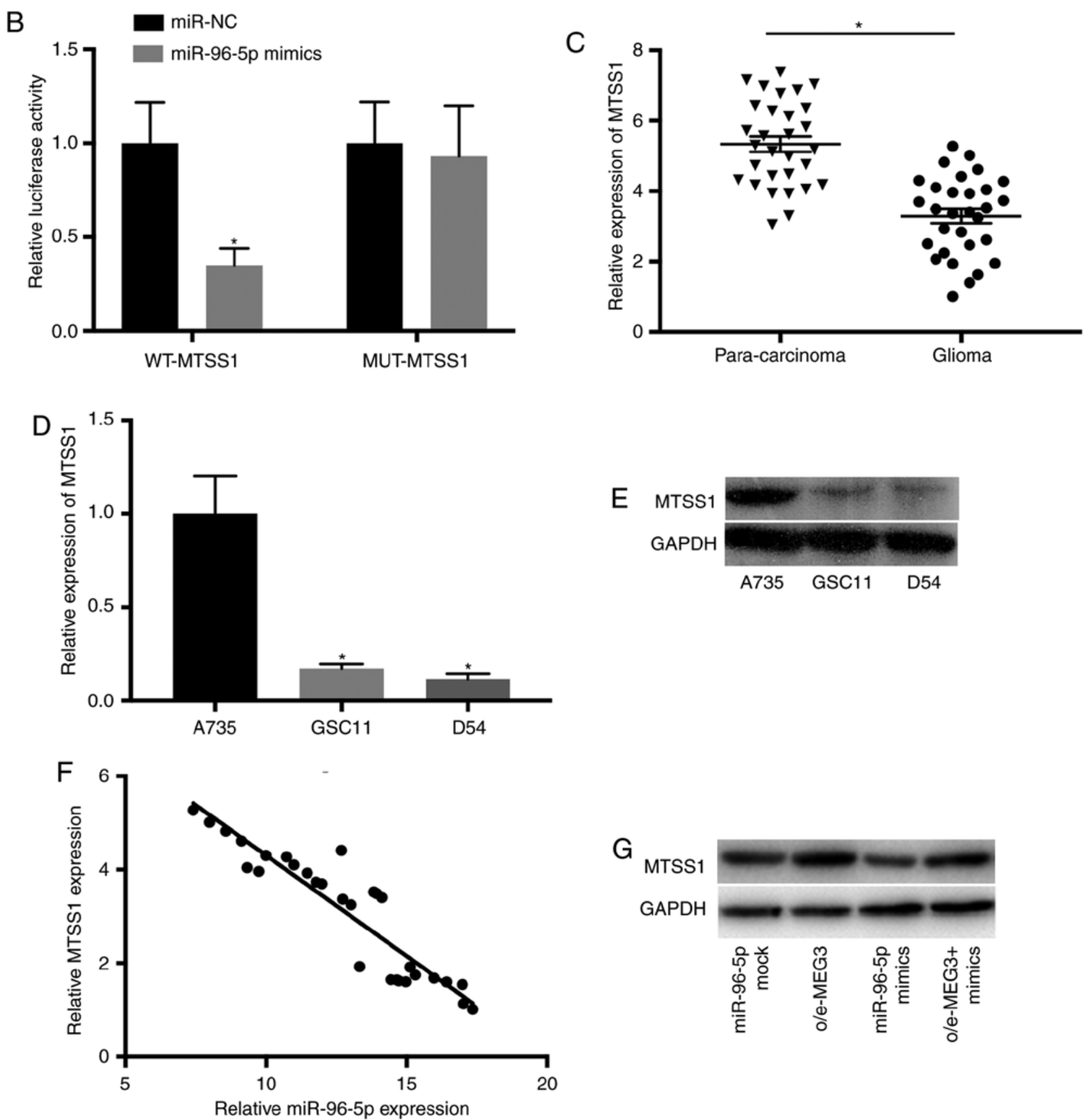

Figure 6. MTSS1 is the putative target of miR-96-5p in glioma. (A) The complementary sequence of miR-96-5p in MTSS1 transcripts was predicted. (B) The association between MTSS1 and miR-96-5p was determined using luciferase assay. "P<0.05 vs. miR-NC. (C) The expression of MTSS1 was downregulated in glioma tissues. " $\mathrm{P}<0.05$. (D and E) The level of MTSS1 was reduced in GSC11 and D54 cells. " $\mathrm{P}<0.05$ vs. A735. (F) MTSS1 and miR-96-5p were negatively correlated in glioma tissues ( $\mathrm{r}=-0.4643 ; \mathrm{P}=0.00931)$. (G) The level of MTSS1 was downregulated by miR-96-5p mimics and upregulated by overexpressed MEG3 in GSC11 cells. miR, microRNA; MTSS1, metastasis suppressor 1.

but not the mutant control (Fig. 6B). In addition, RT-qPCR revealed that the expression of MTSS1 was downregulated in glioma tissues (Fig. 6C). Furthermore, the results of RT-qPCR and northern blotting indicated that MTSS1 was downregulated in GSC11 and D54 cells ( $\mathrm{P}<0.05$ vs. A735; Fig. 6D and E). Additionally, the levels of MTSS1 and miR-96-5p were negatively correlated in glioma tissues (Fig. 6F). 
A previous study revealed that MTSS1 was able to inhibit the migration and invasion of glioma cells by targeting CTTN (35). To further explore MTSS1-mediated mechanisms involved in the development of glioma, the expression of MTSS1 was determined in GSC11 cells transfected with mock control, o/e-MEG3, miR-96-5p mimics or co-transfected with o/e-MEG3 and miR-96-5p mimics. The level of MTSS1 was markedly reduced by miR-96-5p mimics and restored by overexpressed MEG3 in glioma cells (Fig. 6G). These results indicated that MTSS1 is a potential target of miR-96-5p, and MEG3/miR-96-5p/MTSS1 signaling could be associated with the progression of glioma.

\section{Discussion}

Glioma is the most common type of brain cancer in humans, leading to high mortality. Numerous studies have been performed to investigate the pathogenesis of glioma, however, the underlying mechanisms remain largely unknown. Emerging evidence has revealed that IncRNAs are key regulators in the initiation and development of cancer; they function as potential oncogenes or tumor suppressors, and impaired lncRNAs levels could be associated with the progression of tumors (9-16). For example, it was reported that lncRNA PVT1 regulated proliferation, invasion and tumor growth in an orthotopic xenograft generated by breast cancer cells (36). Additionally, downregulated SNHG5 inhibited the growth of gastric cancer via the miR-32/KLF4 signaling pathway (37). Furthermore, lncRNA BC032469 interacted with miR-1207-5p and hTERT, subsequently promoting the proliferation of cancer cells (38). NBAT1 was capable of inhibiting proliferation and promoting apoptosis of ovarian cancer cells (39), and it was also associated with the proliferation and metastasis of glioma cells (40). However, the detailed functions of lncRNAs still require further investigation.

Accumulating evidence has revealed that lncR-MEG3 is a novel tumor suppressor in numerous types of cancer. Downregulation of MEG3 was revealed in cervical cancer, which affects cancer cell growth via miR-21 (41). In addition, the expression of MEG3 was reduced in endometrial carcinoma (19), breast (42) and prostate cancer (43). Downregulated MEG3 was able to promote proliferation of cancer cells, which could be associated with poor prognosis (20,41-43). Consistent with these findings, the present study revealed that MEG3 was significantly downregulated in glioma tissues and cells. Additionally, upregulated MEG3 inhibited the proliferation, migration and invasion of glioma cells.

Previous studies have revealed that various miRNAs are involved in the pathogenesis of glioma $(1,26)$. miRNAs could be putative oncogenic or tumor suppressive factors by acting as key modulators of gene expression, and are important targets of lncRNAs $(32,33)$. Furthermore, miR-93 was able to induce the malignant phenotypes of human glioma cells and lead to chemoresistance (26). In the present study, experiments were carried out to identify the downstream molecules of MEG3 in glioma. The results revealed that MEG3 bound to miR-96-5p, whose expression was significantly upregulated in glioma tissues and cells. In addition, the level of miR-96-5p was suppressed by MEG3 in glioma cells, and their expression levels were negatively correlated in glioma tissues. Furthermore, downregulation of miR-96-5p resulted in inhibited growth and metastasis of glioma. Consistent with the present findings, MEG3 could inhibit the progression of bladder urothelial carcinoma through miR-96 signaling (44). A previous study also revealed that miR-96 promoted transitional cell carcinoma by modulating FOXO1-mediated cell apoptosis (45).

Additionally, MTSS1 was identified as the direct target of miR-96-5p. Further functional study indicated that MTSS1 was downregulated in glioma tissues and cells, and the level of MTSS1 could be decreased by miR-96-5p mimics and restored by overexpressed MEG3, respectively. The expression of MTSS1 has been revealed in numerous tissues such as the prostate, spleen and thymus; however, MTSS1 was downregulated in various types of tumors including gastric, breast and bladder cancer (46-48), which could lead to a poor survival rate. Collectively, the present findings revealed the essential roles of the MEG3/miR-96-5p/MTSS1 axis on the regulation of glioma progression.

In summary, the present study indicated that MEG3 was a promising tumor suppressor that may upregulate MTSS1 through the miR-96-5p pathway, subsequently suppressing the growth and metastasis of glioma. Although previous studies have revealed the functions and downstream pathways of MEG3 in numerous types of cancer including glioma, few studies investigated the interaction between MEG3 and miRNAs $(21,22)$, thus, the present study focused on MEG3-modulated signaling via miRNAs which is relatively novel. The present data revealed the substantial roles of MEG3 and the novel mechanisms underlying the proliferation, migration and invasion of glioma cells, which provided essential evidence on the functions of MEG3 in tumorigenesis. Notably, MEG3/miR-96-5p/MTSS1 signaling could be a putative therapeutic target for the treatment of glioma.

\section{Acknowledgements}

Not applicable.

\section{Funding}

The present study was supported by Liaoning Province Natural Science Fund Program (grant no. 2019-ZD-0835).

\section{Availability of data and materials}

All data generated or analyzed during this study are included in this published article.

\section{Authors' contributions}

WG initiated and designed the present study. SZ and WG performed the experiments and interpreted the results. Both authors read and approved the final manuscript and agree to be accountable for all aspects of the research in ensuring that the accuracy or integrity of any part of the work are appropriately investigated and resolved.

\section{Ethics approval and consent to participate}

The present study was approved by the Ethics Committee of The First Affiliated Hospital of Jinzhou Medical University 
(Jinzhou, China). Written informed consent was obtained from each patient for the use of clinical tissue.

\section{Patient consent for publication}

Not applicable.

\section{Competing interests}

The authors declare that they have no competing interests.

\section{References}

1. Lai NS, Wu DG, Fang XG, Lin YC, Chen SS, Li ZB and Xu SS: Serum microRNA-210 as a potential noninvasive biomarker for the diagnosis and prognosis of glioma. Br J Cancer 112: 1241-1246, 2015.

2. Sathornsumetee S and Rich JN: New treatment strategies for malignant gliomas. Expert Rev Anticancer Ther 6: 1087-1104, 2006.

3. Delgado-López PD and Corrales-García EM: Survival in glioblastoma: A review on the treatment modalities. Clin Trans Oncol 18: 1062-1071, 2016.

4. Gibb EA, Brown CJ and Lam WL: The functional role of long non-coding RNA in human carcinomas. Mol Cancer 10: 38, 2011.

5. Yuan JH, Yang F, Wang F, Ma JZ, Guo YJ, Tao QF, Liu F, Pan W, Wang TT, Zhou CC, et al: A long noncoding RNA activated by TGF- $\beta$ promotes the invasion-metastasis cascade in hepatocellular carcinoma. Cancer Cell 25: 666-681, 2014.

6. Hsiao J, Yuan TY, Tsai MS, Lu CY, Lin YC, Lee ML, Lin SW, Chang FC, Liu Pimentel H, Olive C, et al: Upregulation of haploinsufficient gene expression in the brain by targeting a long non-coding RNA improves seizure phenotype in a model of Dravet syndrome. EBioMedicine 9: 257-277, 2016.

7. Iyer MK, Niknafs YS, Malik R, Singhal U, Sahu A, Hosono Y, Barrette TR, Prensner JR, Evans JR, Zhao S, et al: The landscape of long noncoding RNAs in the human transcriptome. Nat Genet 47: 199-208, 2015.

8. Zhang X, Sun S, Pu JK, Tsang AC, Lee D, Man VO, Lui WM, Wong ST and Leung GK: Long non-coding RNA expression profiles predict clinical phenotypes in glioma. Neurobiol Dis 48 $1-8,2012$

9. Tsang WP, Ng EK, Ng SS, Jin H, Yu J, Sung JJ and Kwok TT: Oncofetal H19-derived miR-675 regulates tumor suppressor RB in human colorectal cancer. Carcinogenesis 31: 350-358, 2010.

10. Wang P, Ren Z and Sun P: Overexpression of the long non-coding RNA MEG3 impairs in vitro glioma cell proliferation. J Cell Biochem 113: 1868-1874, 2012

11. Han L, Zhang K, Shi Z, Zhang J, Zhu J, Zhu S, Zhang A, Jia Z, Wang G, Yu S, et al: LncRNA profile of glioblastoma reveals the potential role of lncRNAs in contributing to glioblastoma pathogenesis. Int J Oncol. 40: 2004-2012, 2012.

12. Zhang XQ, Sun S, Lam KF, Kiang KM, Pu JK, Ho AS, Lui WM, Fung CF, Wong TS and Leung GK: A long non-coding RNA signature in glioblastoma multiforme predicts survival. Neurobiol Dis 58: 123-131, 2013.

13. Zhi F, Wang Q, Xue L, Shao N, Wang R, Deng D, Wang S, Xia X and Yang Y: The use of three long non-coding RNAs as potential prognostic indicators of astrocytoma. PLoS One 10: e0135242, 2015.

14. Vital AL, Tabernero MD, Castrillo A, Rebelo O, Tão H, Gomes F, Nieto AB, Resende Oliveira C, Lopes MC and Orfao A: Gene expression profiles of human glioblastomas are associated with both tumor cytogenetics and histopathology. Neuro Oncol 12: 991-1003, 2010.

15. Zhang X, Zhou Y, Mehta KR, Danila DC, Scolavino S, Johnson SR and Klibanski A: A pituitary-derived MEG3 isoform functions as a growth suppressor in tumor cells. J Clin Endocrinol Metab 88: 5119-5126, 2003.

16. Zhang K, Sun X, Zhou X, Han L, Chen L, Shi Z, Zhang A, Ye M, Wang Q, Liu C, et al: Long non-coding RNA HOTAIR promotes glioblastoma cell cycle progression in an EZH2 dependent manner. Oncotarget 6: 537-546, 2015.

17. Benetatos L, Vartholomatos G and Hatzimichael E: MEG3 imprinted gene contribution in tumourigenesis. Int J Cancer 129: 773-779, 2011.
18. Matouk IJ, Mezan S, Mizrahi A, Ohana P, Abu-Lail R, Fellig Y, Degroot N, Galun E and Hochberg A: The oncofetal H19 RNA connection: Hypoxia, p53 and cancer. Biochim Biophys Acta 1803: 443-451, 2010.

19. Guo Q, Qian Z, Yan D, Li L and Huang L: LncRNA-MEG3 inhibits cell proliferation of endometrial carcinoma by repressing Notch signaling. Biomed Pharmacother 82: 589-594, 2016.

20. Zhou X, Ji G, Ke X, Gu H, Jin W and Zhang G: miR-141 inhibits gastric cancer proliferation by interacting with long noncoding RNA MEG3 and down-regulating E2F3 expression. Dig Dis Sci 60: 3271-3282, 2015.

21. Zhang L, Liang X and Li Y: Long non-coding RNA MEG3 inhibits cell growth of gliomas by targeting miR-93 and inactivating PI3K/AKT pathway. Oncol Rep 38: 2408-2416, 2017.

22. Qin N, Tong GF, Sun LW and Xu XL: Long noncoding RNA MEG3 suppresses glioma cell proliferation, migration and invasion by acting as a competing endogenous RNA of miR-19A. Oncol Rep 25: 1471-1478, 2017.

23. Ma B, Gao Z, Lou J, Zhang H, Yuan Z, Wu Q, Li X and Zhang B: Long non-coding RNA MEG3 contributes to cisplatin-induced apoptosis via inhibition of autophagy in human glioma cells. Mol Med Rep 16: 2946-2952, 2017.

24. Xie H, Fu JL and Xie C: miR-138-5p is downregulated in patients with atrial fibrillation and reverses cardiac fibrotic remodeling via repressing CYP11B2. Eur Rev Med Pharmacol Sci 22: 4642-4647, 2018

25. Zhao X, Sun J, Chen Y, Su W, Shan H, Li Y, Wang Y, Zheng N, Shan $\mathrm{H}$ and Liang $\mathrm{H}$ : LncRNA PFAR promotes lung fibroblast activation and fibrosis by targeting miR-138 to regulate the YAP1-Twist axis. Mol Ther 26: 2206-2217, 2018.

26. Jiang L, Wang C, Lei F, Zhang L, Zhang X, Liu A, Wu G, Zhu J and Song L: miR-93 promotes cell proliferation in gliomas through activation of PI3K/Akt signaling pathway. Oncotarget 6: 8286-8299, 2015

27. Ohta K, Hoshino H, Wang J, Ono S, Iida Y, Hata K, Huang SK, Colquhoun S and Hoon DS: MicroRNA-93 activates c-Met/PI3K/Akt pathway activity in hepatocellular carcinoma by directly inhibiting PTEN and CDKN1A. Oncotarget 6: 3211-3224, 2015

28. Cimmino A, Calin GA, Fabbri M, Iorio MV, Ferracin M, Shimizu M, Wojcik SE, Aqeilan RI, Zupo S, Dono M, et al: miR-15 and miR-16 induce apoptosis by targeting BCL2. Proc Natl Acad Sci USA 102: 13944-13949, 2005.

29. Ke ZP, Xu P, Shi Y and Gao AM: MicroRNA-93 inhibits ischemia-reperfusion induced cardiomyocyte apoptosis by targeting PTEN. Oncotarget 7: 28796-28805, 2016

30. Sullivan TB, Robert LC, Teebagy PA, Morgan SE, Beatty EW, Cicuto BJ, Nowd PK, Rieger-Christ KM and Bryan DJ: Spatiotemporal microRNA profile in peripheral nerve regeneration: miR-138 targets vimentin and inhibits Schwann cell migration and proliferation. Neural Regen Res 13: 1253-1262, 2018.

31. Liang Z, Feng Q, Xu L, Li S and Zhou L: CREPT regulated by miR-138 promotes breast cancer progression. Biochem Biophys Res Commun 493: 263-269, 2017.

32. Zhao H, Peng R, Liu Q, Liu D, Du P, Yuan J, Peng G, Liao Y: The lncRNA H19 interacts with miR-140 to modulate glioma growth by targeting iASPP. Arch Biochem Biophys 610: 1-7, 2016.

33. Chen L, Wang Y, He J, Zhang C, Chen J and Shi D: Long non-coding RNA H19 promotes proliferation and invasion in human glioma cells by downregulating miR-152. Oncol Res: Feb 8, 2018 doi: 10.3727/096504018X15178768577951 (Epub ahead of print).

34. Livak KJ and Schmittgen TD: Analysis of relative gene expression data using real-time quantitative PCR and the 2-Delta Delta C(T)) method. Methods 25: 402-408, 2001.

35. Zhang S and Qi Q: MTSS1 suppresses cell migration and invasion by targeting CTTN in glioblastoma. J Neurooncol 121: 425-431, 2015.

36. Tang J, Li Y, Sang Y, Yu B, Lv D, Zhang W and Feng H: LncRNA PVT1 regulates triple-negative breast cancer through KLF5/beta-catenin signaling. Oncogene 37: 4723-4734, 2018.

37. Zhao L, Han T, Li Y, Sun J, Zhang S, Liu Y, Shan B, Zheng D and Shi J: The lncRNA SNHG5/miR-32 axis regulates gastric cancer cell proliferation and migration by targeting KLF4. FASEB J 31: 893-903, 2017.

38. Lü MH, Tang B, Zeng S, Hu CJ, Xie R, Wu YY, Wang SM, He FT and Yang SM: Long noncoding RNA BC032469, a novel competing endogenous RNA, upregulates hTERT expression by sponging miR-1207-5p and promotes proliferation in gastric cancer. Oncogene 35: 3524-3534, 2016. 
39. Yan C, Jiang Y, Wan Y, Zhang L, Liu J, Zhou S and Cheng W: Long noncoding RNA NBAT-1 suppresses tumourigenesis and predicts favorable prognosis in ovarian cancer. Onco Targets Ther 10: 1993-2002, 2017.

40. Pandey GK, Mitra S, Subhash S, Hertwig F, Kanduri M, Mishra K, Fransson S, Ganeshram A, Mondal T, Bandaru S, et al: The risk-associated long noncoding RNA NBAT-1 controls neuroblastoma progression by regulating cell proliferation and neuronal differentiation. Cancer Cell 26: 722-737, 2014.

41. Zhang J, Yao T, Wang Y, Yu J, Liu Y and Lin Z: Long noncoding RNA MEG3 is downregulated in cervical cancer and affects cell proliferation and apoptosis by regulating miR-21. Cancer Biol Ther 17: 104-113, 2016.

42. Zhang J, Guo S and Jia B: Down-regulation of long non-coding RNA MEG3 serves as an unfavorable risk factor for survival of patients with breast cancer. Eur Rev Med Pharmacol Sci 20 5143-5147, 2016.

43. Luo G, Wang M, Wu X, Tao D, Xiao X, Wang L, Min F, Zeng F and Jiang G: Long non-coding RNA MEG3 inhibits cell proliferation and induces apoptosis in prostate cancer. Cell Physiol Biochem 37: 2209-2220, 2015.
44. Liu G, Zhao X, Zhou J, Cheng X, Ye Z and Ji Z: Long non-coding RNA MEG3 suppresses the development of bladder urothelial carcinoma by regulating miR-96 and TPM1. Cancer Biol Ther 19: 1039-1056, 2018.

45. Guo Y, Liu H, Zhang H, Shang $\mathrm{C}$ and Song Y: miR-96 regulates FOXO1-mediated cell apoptosis in bladder cancer. Oncol Lett 4: $561-565,2012$.

46. Lee YG, Macoska JA, Korenchuk S, and Pienta K: MIM, a potential metastasis suppressor gene in bladder cancer. Neoplasia 4: 291-294, 2002.

47. Liu K, Wang G, Ding $\mathrm{H}$, Chen $\mathrm{Y}, \mathrm{Yu}$ G, and Wang J: Downregulation of metastasis suppressor 1 (MTSS1) is associated with nodal metastasis and poor outcome in Chinese patients with gastric cancer. BMC Cancer 10, 428, 2010.

48. Parr C and Jiang WG: Metastasis suppressor 1 (MTSS1) demonstrates prognostic value and anti-metastatic properties in breast cancer. Eur J Cancer 45: 1673-1683, 2009.

(c) (i) () This work is licensed under a Creative Common International (CC BY-NC-ND 4.0) License. 